\title{
Optimization of SVC settings to improve post-fault voltage recovery and angular stability
}

\author{
Weihong HUANG ${ }^{1}$, Kai SUN $^{1}$ [D
}

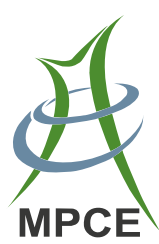

\begin{abstract}
In this study, controller parameters of static var compensators (SVCs) at planned locations are optimized to mitigate fault-induced delayed voltage recovery issues and improve angular stability of a multi-machine power system. The problem is formulated as a nonlinear optimization problem involving constraints on post-fault trajectories of voltages and frequencies. This paper proposes a mesh adaptive direct search based algorithm interfaced with a power system simulator for optimization of SVC controller parameters. The proposed method is tested on the NPCC 140-bus system to optimize controller parameters for three SVCs. Simulations on critical contingencies verify that post-fault transient voltages and generator speeds can both quickly recover and transient stability of the system is improved.
\end{abstract}

Keywords Static var compensator (SVC), Fault induced delayed voltage recover issue (FIDVR), Angular stability, Mesh adaptive direct search (MADS) algorithm

CrossCheck date: 24 August 2018

Received: 20 December 2017/ Accepted: 24 August 2018/Published online: 17 December 2018

(c) The Author(s) 2018

$\triangle$ Kai SUN

kaisun@utk.edu

Weihong HUANG

whuang@utk.edu

1 The Department of Electrical Engineering and Computer Science, The University of Tennessee, Knoxville, USA

\section{Introduction}

The primary application of static var compensators (SVCs) in a power system is to maintain bus voltages and provide reactive power support. An SVC is a fast acting power controller device, which also can introduce new flexibility into the operations of a power system. For example, an SVC can improve angular stability with a damping controller installed [1]. This extra flexibility permits the SVC to enhance varying operational conditions and improve the use of existing installations. This paper aims at optimizing the controller parameters of SVCs to mitigate fault induced delayed voltage recovery (FIDVR) issues and, simultaneously, to improve angular stability when subjecting to a severe disturbance.

Several papers have studied the optimal allocation of dynamic var sources such as SVCs and static synchronous compensator (STATCOMs) to addressing FIDVR issues in [2-14]. References [2-6] mainly focus on the placement of dynamic var sources considering short-term instability. References [7-14] optimize the sizes of the dynamic var sources at predetermined locations.

Similarly, many papers have been published in the field of designing dynamic var source controllers for power system stability improvements [15-23]. References [15-20] require linearization of the system as they are based on small disturbance analysis or optimize the SVC parameters with linearized model and check with dynamic simulation once merely for verification. References [21-23] consider the dynamics of generator speeds that capture complex dynamics of system, especially during major disturbance.

Most of existing methods proposed in the aforementioned references optimize the allocation and settings of SVCs to improve either post-fault dynamic voltage 
recovery or angular stability. Few methods have been proposed to optimize SVCs addressing the dynamic recoveries of both bus voltages and generator speeds following a fault.

The main difficulties of the optimal controller parameters of dynamic var sources considering power system dynamics lie in two aspects. First, the problem of the optimization of controller parameters is a nonlinear, nonconvex optimization problem under high dimensional computation complex. Second, checking the constraints on FIDVR and generator speeds requires the post-fault power system trajectories, which can only be obtained from accurate power system differential-algebraic equation (DAE) models by, e.g., a power system time-domain simulation. Thus, both a nonlinear optimization solver and a power system DAE solver are needed and should be integrated by an efficient interfacing algorithm.

In order to tackle this problem at a large scale, one solution is the blackbox optimization, which makes the simulation and constraints checking be a blackbox providing an output for any specified value of system inputs, i.e. the controller parameters of dynamic var sources for this case. The mesh adaptive direct search (MADS) algorithm is one of useful blackbox optimization algorithms $[24,25]$. The MADS algorithm is a frame-based and derivative-free method under general nonlinear constraints. The MADS algorithm performs well on optimizations based on the result of expensive computer simulations in which the gradient information may not be available, contaminated noise can exist, or feasible solutions are not easy to find. The MADS algorithm has been proved effective in solving some power system optimization problems involving DAEs in recent studies: for example, the optimal PMU placement for power system dynamic state estimation based on empirical observability gramian [26], the optimal placement of dynamic var sources based on empirical controllability covariance [6], and the optimal sizes of dynamic var sources addressing FIDVR issue [27].

In this paper, five parameters of the leading-lag controllers with SVCs are optimized by an MADS-based method. Unlike [27], the SVCs are assumed to be placed at predetermined locations and have predetermined sizes to ensure post-fault fast voltage recovery. The optimization on the SVC control settings in this paper will aim at improving angular stability while still avoiding FIDVR issues. Adding angular stability improvement to the objective function will largely increase the computational complex of the optimization problem since the parameters of the SVC controller will need to be optimized. Also, simulated waveforms of generator speeds need to be recorded to check for damping ratios in addition to all existing constraints for the FIDVR issues in [27].
The optimization is conducted by integrating a power system time-domain simulation tool with an MADS solver under the framework of blackbox optimization [27]. The proposed approach is validated on the NPCC system representing the northeast region of the U.S. Eastern Interconnection. The five controller parameters of three SVCs planned in the NPCC are optimized for mitigating FIDVR issues as well as improving angular stability under given critical contingencies. The proposed approach can quickly converge to the optimal solution after a small number of iterative evaluations.

The rest of this paper is organized as follows. Section 2 discusses the formulation of the problem. Section 3 introduces the MADS algorithm. Section 4 proposes an MADS based approach to optimize controller parameters of SVCs. Then in Section 5, the proposed approach is validated on the NPCC 140-bus system. Finally, the conclusion is drawn in Section 6.

\section{Formulation of the problem}

Assume that the buses to install SVCs and their sizes have been determined by power system planners using an optimal allocation approach as well as engineering judgement-giving considerations to factors on power system operations. The aim of the optimization problem in present paper is to minimize the average of absolute speed deviations of all generators (whose index set is denoted by $I_{\mathrm{G}}$ ) in order to improve angular stability in the system from the time of fault clearing $t_{c l}$ to the end of simulation $t_{e n d}$. At the same time, the system should satisfy the system dynamic constraints for $K$ selected contingencies, i.e., no FIDVR issue. This optimization problem can be formulated as (1)(8), where the physical meaning of the objective function is a weighted average of generator speed deviations over the concerned post-fault period.

$$
\min J=\frac{\Delta t}{K\left|I_{\mathrm{G}}\right|\left(t_{\text {end }}-t_{c l}\right)} \sum_{k=1}^{K} \sum_{i=1}^{\left|I_{\mathrm{G}}\right|} \sum_{t=t_{c l}}^{t_{\text {end }}}\left|\Delta \omega_{k, i}(t, \boldsymbol{Y})\right| \Delta t
$$

s.t.

$\boldsymbol{Y}_{\mathrm{L}} \leq \boldsymbol{Y} \leq \boldsymbol{Y}_{\mathrm{U}}$

$\dot{\boldsymbol{x}}=\boldsymbol{f}(\boldsymbol{x}, \boldsymbol{V}, \boldsymbol{Y})$

$\mathbf{0}=\boldsymbol{g}(\boldsymbol{x}, \boldsymbol{V}, \boldsymbol{Y})$

$\left|\frac{V_{I_{\mathrm{G}}, k}(t, \boldsymbol{Y})-V_{I_{\mathrm{G}}, \text { init }}}{V_{I_{\mathrm{G}}, \text { init }}}\right| \leq S_{1} \quad t \in\left[t_{c l}, t_{s}\right]$

$\left|\frac{V_{I_{\mathrm{L}}, k}(t, \boldsymbol{Y})-V_{I_{\mathrm{L}}, \text { init }}}{V_{I_{\mathrm{L}}, \text { init }}}\right| \leq S_{2} \quad t \in\left[t_{c l}, t_{s}\right]$ 
$\left|\frac{V_{I_{\mathrm{L}}, k}(t, \boldsymbol{Y})-V_{I_{\mathrm{L}}, \text { init }}}{V_{I_{\mathrm{L}}, \text { init }}}\right| \geq S_{3} \quad t \in\left[t_{c l}, t_{s}\right]$

$\left|\frac{V_{N, k}(t, \boldsymbol{Y})-V_{N, \text { init }}}{V_{N, \text { init }}}\right| \leq S_{4} \quad t \in\left[t_{s}, t_{\text {end }}\right]$

where $\Delta \omega_{k, i}(t, \boldsymbol{Y})$ is the speed deviation of the $i$ th generator under $k$ th contingency for controller parameters $\boldsymbol{Y}$ of SVCs at time $t ; \boldsymbol{Y}=\left[Y_{1}, Y_{2}, \ldots, Y_{R}\right]^{\mathrm{T}}$ is a column vector about the $r$ th dynamic var controller parameters, whose upper and lower limits are respectively in vectors $\boldsymbol{Y}_{\mathrm{L}}$ and $\boldsymbol{Y}_{\mathrm{U}}$. Equations (3) and (4) represent the DAEs for post-fault power system trajectories, $\boldsymbol{x}$ is the state vector, and $\boldsymbol{V}$ is a vector of all voltage magnitudes. Equations (5)-(8) represent the dynamic constraints about post-fault voltage recovery performances. $I_{\mathrm{G}}$ indicates generator buses, $I_{\mathrm{L}}$ indicates load buses, $N$ indicates the total number of all buses in a system, $V_{I_{\mathrm{G}}, k}(t, \boldsymbol{Y})$ is the voltage of generator bus under $k$ th contingency at time $t$ for the controller parameters $Y, T_{\max }$ is the maximum time duration, $S_{1}$ to $S_{4}$ are the voltage deviation percentages, $t_{s}$ is the end time of the transient period, and $\Delta t$ is simulation time step.

Based on industry practices, NERC/WECC standards [28] are considered in (5)-(8) for $N-1$ cases: $S_{1}=30 \%$, $S_{2}=25 \%, S_{3}=20 \%, S_{4}=5 \%$, and $T_{\max }=20$ cycles; $t_{s}$ is $3 \mathrm{~s}$ after the $t_{c l}$. For load buses (whose index set is denoted by $I_{\mathrm{L}}$ ), the post-fault voltage dynamic constraints are illustrated in (6)-(8), where (6) limits the maximum transient voltage overshoot or dip; (7) is about the duration of a transient voltage sag; (8) concerns voltage deviations in the post-transient period. To largely ensure effectiveness and robustness of controller parameters, additional $N-1$ or $N-1-1$ contingencies in the system as well as different operating conditions could be considered in (3)-(8).

The optimization is coupled with resolution of power system DAEs in (3), (4) as well as dynamic constraints in (5)-(8), which dramatically increase the computational complexities and make the feasible solutions of the nonlinear optimization problem nonconvex. In addition, no gradient information can easily be obtained and used. Therefore, an idea to deal with this complex problem is to use black optimization, which takes the simulation tool, checking of post-fault voltage recovery constraints, obtaining speed deviations of generators, and calculating the objective function as a black box.

The blackbox optimization approach to solve this problem is illustrated in Fig. 1. The blackbox utilizes a power system simulator to obtain the post-fault trajectories of $\boldsymbol{V}$ and $\boldsymbol{\omega}$ under contingency $k$, makes use a verification model to check all criteria for $\boldsymbol{V}$, and evaluates the objective function $J$. The input of the blackbox is $\boldsymbol{Y}$, and the output is the objective function value $J(\boldsymbol{Y})$. Based on the outputs from the blackbox, the optimization algorithm

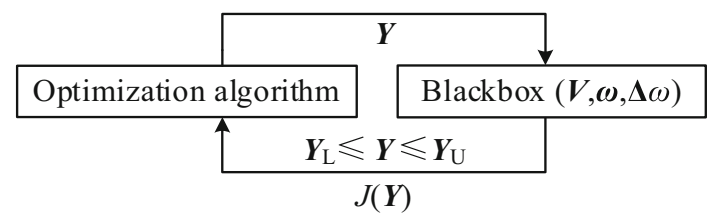

Fig. 1 Schematic procedure of blackbox optimization for dynamic var controller parameters setting problem

iteratively updates the input $\boldsymbol{Y}$ of the blackbox until a stopping criterion is satisfied.

To deal with simulation based derivative-free blackbox function, the MADS algorithm is targeted as the optimization algorithm in this paper. In the following two sections, the MADS algorithm is introduced by illustrating an optimization on a two-dimensional non-convex function, and then, an MADS algorithm based approach is proposed to solve the problem of optimal controller parameters for SVCs.

\section{Introduction of MADS algorithm}

The MADS algorithm is an effective iterative optimization in dealing with general nonlinear nonsmooth constraints, such as a simulation based blackbox functions [28]. Meanwhile, it aims at converging to the best solution with a small number of evaluation at some trial points.

Here, a two-dimensional non-convex nonlinear McCormick function is illustrated how a derivative-free function converges to optimum by the MADS algorithm. The McCormick is often used as a benchmark problem for testing the performances of optimization problems [28], which has two local minima. In Fig. 2, the global optimum solution of McCormick function is marked by a four-point star and the smaller the objective function, the colder the color.

In each iteration, the MADS conducts three steps, i.e. poll, search, and update. As shown in Fig. 2a for iteration $b$, first, it evaluates the blackbox function at trial point $o_{b}$. Then, in the search step, it generates thin lines representing the mesh with size $\Delta_{b}^{m}$. After that, it conducts polls step on a mesh by thick lines with size $\Delta_{b}^{p}$, where $\Delta_{b}^{p}$ is equal to $\Delta_{b}^{m}$ in this iteration, and evaluates all the polls, i.e. $a_{1}, a_{2}$, and $a_{3}$. When the three trial points fail to find a better solution comparing to the point $o_{b}, o_{b+1}$ stays at $o_{b}$ and all visited points $\left(a_{1}, a_{2}\right.$, and $\left.a_{3}\right)$ are updated in a set which avoids duplicated trial points in iterations.

The MADS algorithm is continued at iteration $b+1$ in Fig. 2b. Search mesh size $\Delta_{b+1}^{m}$ is reduced to $1 / 4$ and poll size $\Delta_{b+1}^{p}$ is reduced to $1 / 2$ with trial points $a_{4}, a_{5}$, and $a_{6}$. These trial points successfully find a better solution at $a_{4}$, 


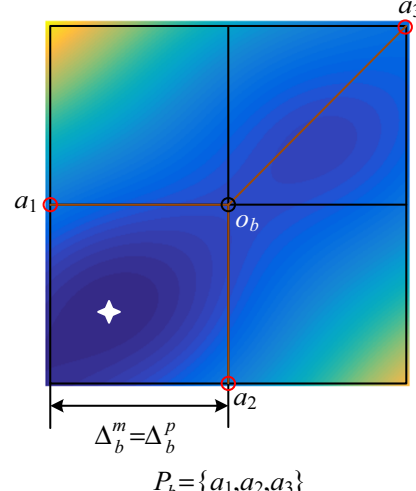

$P_{b}=\left\{a_{1}, a_{2}, a_{3}\right\}$

(a) At iteration $b$

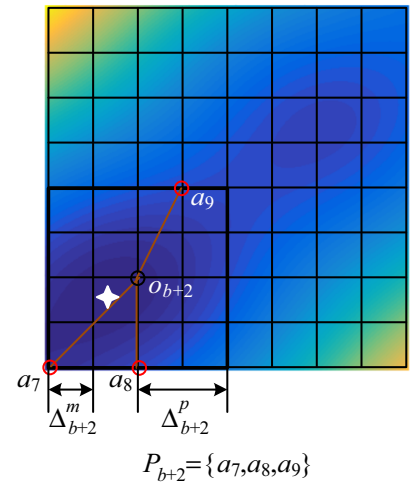

(c) At iteration $b+2$

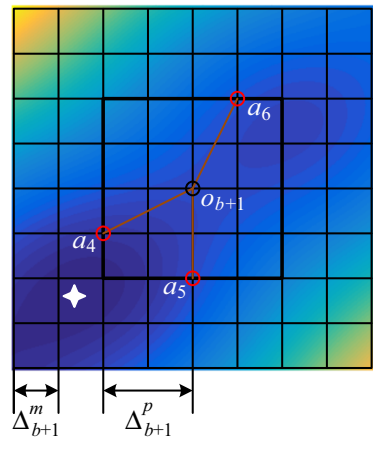

$P_{b+1}=\left\{a_{4}, a_{5}, a_{6}\right\}$

(b) At iteration $b+1$

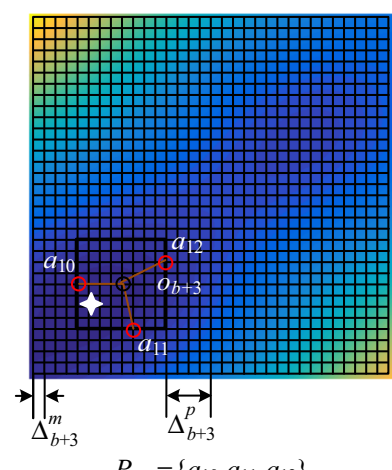

$P_{b+3}=\left\{a_{10}, a_{11}, a_{12}\right\}$

(d) At iteration $b+3$
Fig. 2 MADS algorithm based blackbox optimization

so at iteration $b+2, \Delta_{b+2}^{m}$ and $\Delta_{b+2}^{p}$ keep still, and the $o_{b+2}$ moves to the $a_{4}$ in Fig. 2c. Similarly, three trial points fail to find a better solution at $a_{7}, a_{8}, a_{9}$, so $\Delta_{b+3}^{m}$ is reduced to $1 / 16$ which is faster than the decrease of $\Delta_{b+3}^{p}=1 / 4$, and the number of candidate poll locations increases shown as Fig. 2d. Then, a better solution is successfully found among three trial points at $\alpha_{10}$, the poll and search size keep still in the next step. Iteratively, do the search and poll in the searching space by refining of meshes, the solution reaches to the optimal until meet the stop criterion.

In other words, for each iteration, if in the poll step, it fails to find an improved mesh point, $o_{b+1}$ stays at previous, shrink the mesh $\Delta_{b}^{m}$ and $\Delta_{b}^{p}$; otherwise, $o_{b+1}$ move to the new $o_{b+1}, \Delta_{b}^{p}$ and $\Delta_{b}^{m}$ keep still. Finally, update the all evaluated points in the set.

\section{Proposed MADS-based approach}

The proposed MADS-based approach for optimizing the controller parameters of SVCs integrates power system simulation using (3) and (4), NERC/WECC voltage constraints checking modules for (5)-(8), and evaluation of objective function (1) in a blackbox. A power system DAE solver (e.g. PowerTech DSA tool TSAT) is interfaced with the MADS algorithm: DAEs (3) and (4) are solved to obtain the post-fault voltage responses of all buses $\boldsymbol{V}$ and speed responses of generators $\omega$; the results of the checking of voltage criteria in constraints and objective function of speed deviations are sent to the MADS algorithm for a control strategy.

Specifically, the following steps are included:

Step 1: Predetermine the sites and sizes of SVCs, which are usually solved separately. First, the siting problem can be solved by calculating and comparing voltage sensitivity indices [7] or empirical controllability covariance [6] at candidate locations. Then, the sizes of SVCs can be optimized by heuristic searching algorithms interfacing with a power system simulator, such as Voronoi diagram method integrating linear programming in [29]. The factors considered in the siting and sizing optimization of SVCs can be land prices, installation fees, maintenance costs, and penalties of violation of voltage recovery criteria.

Step 2: Set initial controller parameters $\boldsymbol{Y}$ for SVCs and boundary of their upper and lower limits. Once the siting and sizing of SVCs are determined, initial controller parameters of SVCs could be from either default values [29] or random setting within the required range [30].

Step 3: Perform the search and poll steps of the MADS algorithm, and pass the $\boldsymbol{Y}$ to the blackbox.

Step 4: The blackbox receives $\boldsymbol{Y}$ from the MADS algorithm and uses $\boldsymbol{Y}$ as new controller parameters of SVCs to simulate the post-fault power system trajectories. Then, all voltage trajectories $\boldsymbol{V}$ are checked with the criteria (5)-(8), and the speed deviations of generators $\omega$ are recorded. Later, the objective function is evaluated by calculating $\Delta \omega$, and the results including $J$ and constraints are feed back to the MADS algorithm.

Step 5: Determine the mesh point by considering the evaluated settings and objective functions in the MADS algorithm. If it fails to find an improved mesh point $o_{b+1}$, stay with previous $o_{b+1}=o_{b}$, shrink the mesh $\Delta_{b+1}^{m}$ and update $\Delta_{b+1}^{p}$; otherwise, jump to the new $o_{b+1}$, stay with previous $\Delta_{b+1}^{p}, \Delta_{b+1}^{m}$. Then, update the previous evaluated setting in the set.

Step 6: Check the stopping criterion: the total number of iterations $b$ exceeds a certain number or $\Delta_{b+1}^{p}$ is less than a certain value indicating a convergence of the tentative optimum. If it is met, stop the procedure; otherwise, go back to Step 3. 


\section{Case study}

The proposed approach is tested on the NPCC 48-machine 140-bus system, which represents a simplified equivalent of the northeast region of the Eastern Interconnection power grid in the U.S. and Canada, including control areas of six New England States, New York State, Ontario, Québec, New Brunswick, and Nova Scotia. Particularly, the power system of six New England states is the well-known IEEE 39-bus test system. The simulation period for each contingency is $5 \mathrm{~s}$ starting from the fault. The integration time step $\Delta t$ is $1 / 240 \mathrm{~s}$. It is true that different time windows may result into different optimization results. For our problem, the first 1 to 3 swings are most critical to check criteria on bus voltages as well as speed deviations of generators, and for this system, the voltage and speed waveforms basically settle down in 5 s. If a longer time window is set, the proposed method can still work but the computation time for DAEs have to be longer. The power system DAE solver adopts software TSAT by Powertech Labs, and the MADS algorithm is implemented by the NOMAD (nonlinear optimization by MADS in MATLAB) solver [24].

\subsection{SVC and load modeling}

In time-domain simulation, dynamic load model is crucial to simulate the phenomena of FIDVR and SVC modelling is important for the performance of dynamic var support. In the case study, each load adopts the PSS/E CLODBL load model [29], which contains a large motor, a small motor, a lighting load, and a ZIP load. Each SVC adopts the CSVGN5 model of PSS/E. Main parameters of

Table 1 Parameters for SVC model

\begin{tabular}{lll}
\hline Parameters & Range & Value \\
\hline Filter lag time constant $T_{\mathrm{S} 1}$ & $<0.4 \mathrm{~s}$ & 0 \\
Voltage error maximum $V_{\mathrm{e}, \max }$ & $<0.3$ & 0.15 \\
$1^{\text {st }}$ stage lead time constant $T_{\mathrm{S} 2}$ & $<2 \mathrm{~s}$ & $0.1 \mathrm{~s}$ \\
$1^{\text {st }}$ stage lag time constant $T_{\mathrm{S} 3}$ & $(0,5 \mathrm{~s})$ & $4.5 \mathrm{~s}$ \\
$2^{\text {nd }}$ stage lead time constant $T_{\mathrm{S} 4}$ & $<2 \mathrm{~s}$ & 0 \\
$2^{\text {nd }}$ stage lag time constant $T_{\mathrm{S} 5}$ & $<5 \mathrm{~s}$ & 0 \\
Gain $K_{\mathrm{Svs}}$ & $(50,1000)$ & 50 \\
Time constant $K_{\mathrm{SD}}$ & $(0,1000 \mathrm{~s})$ & 0 \\
Maximum susceptance of SVC $B_{\max }$ & $(0,10)$ & 1 \\
Threshold upper limit $B_{\max }{ }^{\prime}$ & $<B_{\max }$ & 0.9 \\
Threshold lower limit $B_{\min }{ }^{\prime}$ & $>-2 B_{\max }$ & -0.9 \\
Minimum susceptance of SVC $B_{\min }$ & $<B_{\min }{ }^{\prime}$ & -1 \\
Thyristor delay $T_{\mathrm{S} 6}$ & $(2 \Delta t, 0.2 \mathrm{~s})$ & $0.05 \mathrm{~s}$ \\
Deviation voltage & $(0,0.5)$ & 0.15 \\
\hline
\end{tabular}

the SVC model in the case studies are listed in the Table 1 [30]. The second column of the table has suggested ranges of values from [30] and the third column gives the default settings used in our case studies, which are from [29]. Particularly, the lead-lag damping controller consists of 2 lead-lag compensators. The five parameters of the that controller, including four time constants $T_{\mathrm{S} 1}$ to $T_{\mathrm{S} 4}$ and one gain $K_{\mathrm{SVS}}$, are those to be optimized in this paper for mitigation of FIDVR issues and enhancement of angular stability. The optimized controller parameters aim to help control actual var outputs accurately at desired values with minimized delays or overshoots.

\subsection{NPCC system without SVCs}

The NPCC 140-bus system is shown in Fig. 3. According to contingency analysis, a three-phase fault on bus 6 , which is cleared by the line trip between bus 6 and bus 7 after 5 cycles, is identified to be the most severe one among all " $N-1$ " contingencies. In the following, the SVC controllers will be optimized against this most severe contingency.

Figure 4 shows bus voltages from time domain simulation without SVCs, where FIDVR occurs due to these violations: (1) some load bus voltages are below $75 \%$ of the initial voltages; (2) some buses violate the constraint (7) because the durations of their voltage dips between $20 \%$ and $25 \%$ of initial voltages are more than 20 cycles; (3) several buses violate constraint (8) because their voltage deviations are higher than $5 \%$ of initial voltages in the post transient period.

Figure 5 shows the speeds of generators 47, 91, 130 and 139 in time domain simulation without SVCs. The average speed deviations of all generators is $0.0704 \mathrm{~Hz}$. The system has significant oscillations; generator 139 is the most vulnerable one and may potentially go out of step from the other generators in the system.

\subsection{NPCC system with SVCs}

In order to mitigate the above FIDVR issue, three SVCs of 400 Mvar, 400 Mvar, and 255 Mvar are installed at three predetermined buses 3, 6, and 34, respectively. The locations and sizes of SVCs are respectively optimized by the empirical controllability covariance based fault specified method in [6] and the Voronoi diagram based method in [29].

First, the damping controller parameters of the SVCs are set with default values as in [29], and the resulting voltages are shown in Fig. 6 without FIDVR. Although the postfault voltage trajectory of a load bus is lower than $80 \%$ of its initial value, the duration between $75 \%$ and $80 \%$ is less than 10 cycles. With the default controller parameters, the 


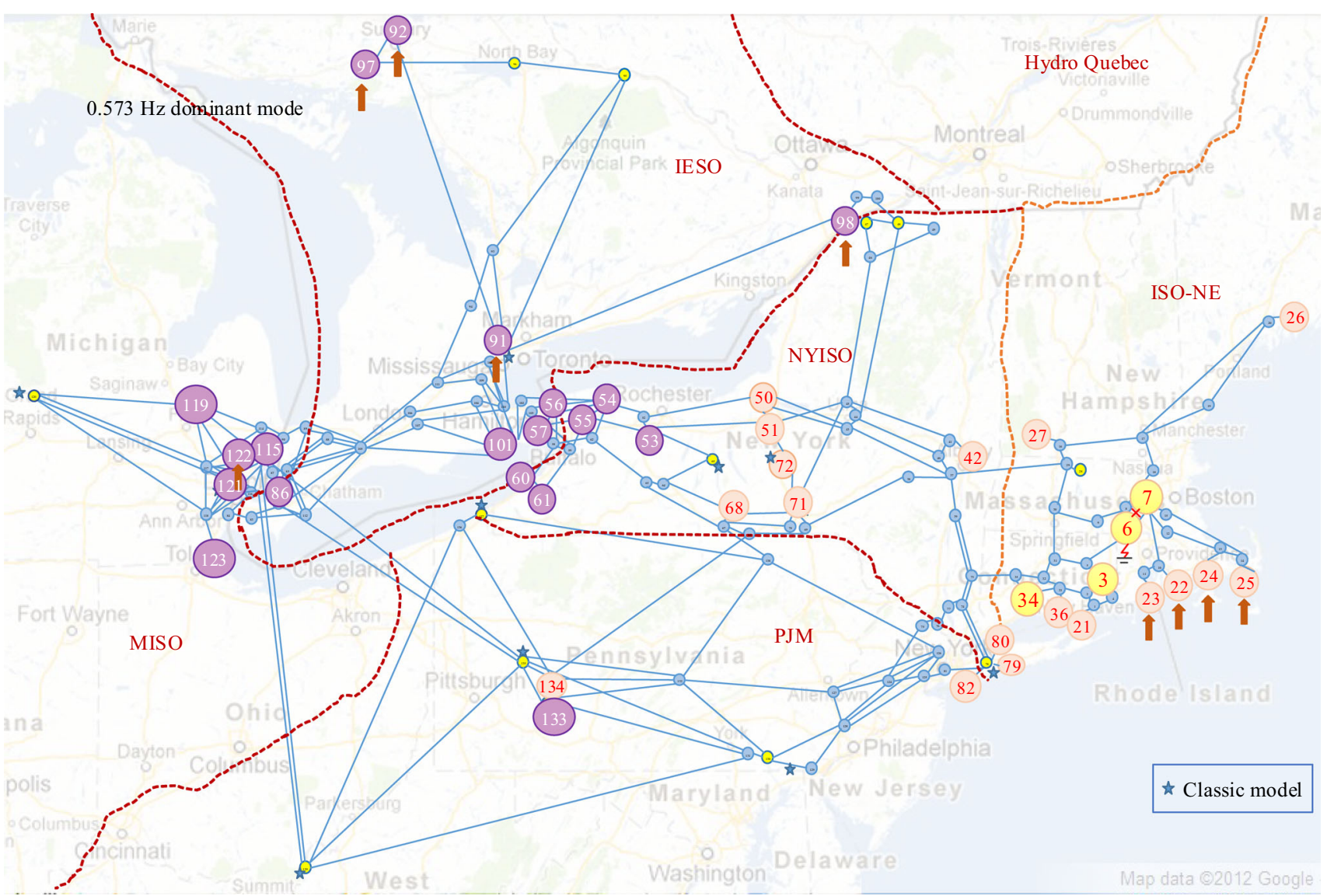

Fig. 3 NPCC system and the most severe $N-1$ contingency and three candidate buses for SVCs with predetermined sizes

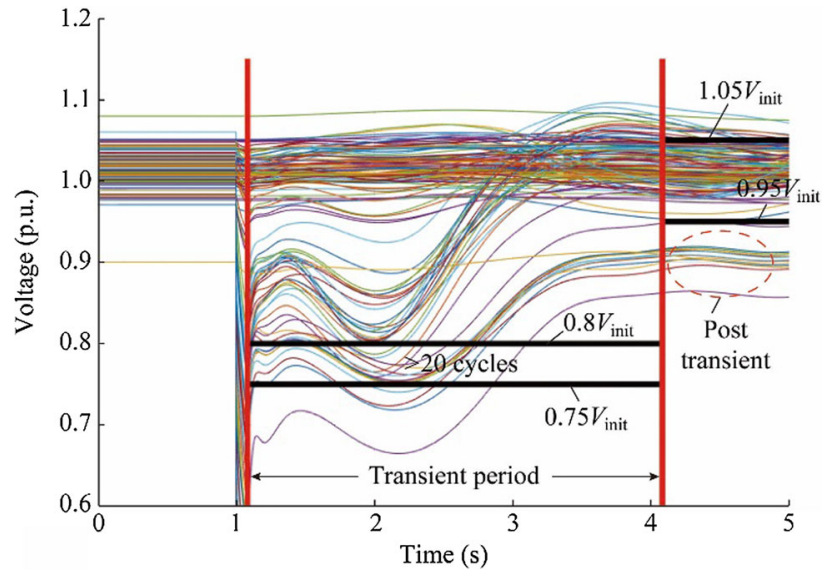

Fig. 4 Voltage responses without SVC support

objective function, i.e. the average speed deviation during the post-fault period for all generators in the system as (1) is reduced to $0.0452 \mathrm{~Hz}$.

Second, the same contingency is considered, and the five SVC controller parameters are optimized and applied to all three SVCs. The optimal parameters are $\left[T_{\mathrm{S} 2}, T_{\mathrm{S} 3}, T_{\mathrm{S} 4}, T_{\mathrm{S} 5}\right.$, $\left.K_{\mathrm{SVS}}\right]=[0.17 \mathrm{~s}, 1.19 \mathrm{~s}, 0.17 \mathrm{~s}, 1.27 \mathrm{~s}, 1000]$ for buses 3,6

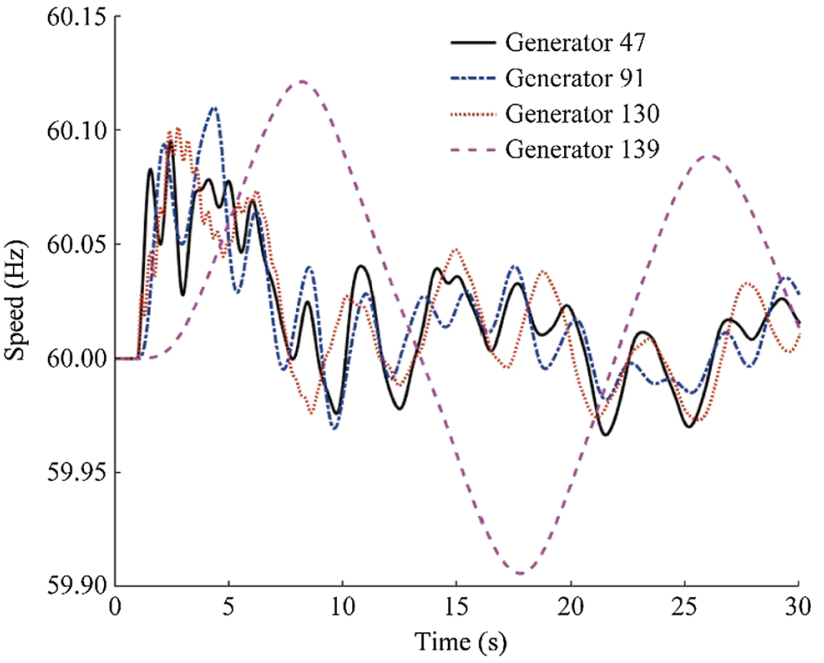

Fig. 5 Speeds of typical generators without SVC support

and 34. The average speed deviation for all generators is reduced from $0.0452 \mathrm{~Hz}$ to $0.0418 \mathrm{~Hz}$.

Finally, the controller parameters $\left[T_{\mathrm{S} 2}, T_{\mathrm{S} 3}, T_{\mathrm{S} 4}, T_{\mathrm{S} 5}\right.$, $\left.K_{\mathrm{SVS}}\right]$ for three SVCs are respectively optimized, which turn out to be respectively equal to $[0,4.30 \mathrm{~s}, 0.11 \mathrm{~s}, 2.39 \mathrm{~s}$, 1000] at bus $3,[0.51 \mathrm{~s}, 3.47 \mathrm{~s}, 0.06 \mathrm{~s}, 1.83 \mathrm{~s}, 951]$ at bus 6 


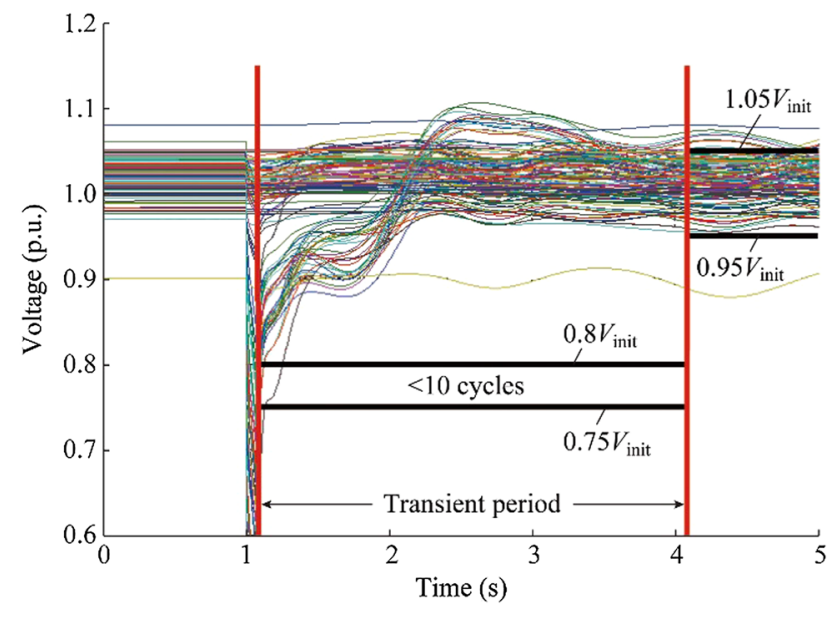

Fig. 6 Voltage response with SVC support

Table 2 Comparison of objective functions

\begin{tabular}{ll}
\hline Channels & Objective function $J(\boldsymbol{Y})(\mathrm{Hz})$ \\
\hline No SVC & 0.0704 \\
SVC-default & 0.0452 \\
SVC-1 OPT & 0.0418 \\
SVC-3 OPT & 0.0406 \\
\hline
\end{tabular}

Table 3 Comparison of damping ratios

\begin{tabular}{lllll}
\hline Modes (Hz) & \multicolumn{4}{l}{ Damping ratio (\%) } \\
\cline { 2 - 5 } & $\begin{array}{l}\text { No } \\
\text { SVC }\end{array}$ & $\begin{array}{l}\text { SVC- } \\
\text { default }\end{array}$ & $\begin{array}{l}\text { SVC-1 } \\
\text { OPT }\end{array}$ & \multicolumn{1}{l}{$\begin{array}{l}\text { SVC-3 } \\
\text { OPT }\end{array}$} \\
\hline 0.573 & 3.229 & 7.337 & 8.406 & 11.088 \\
$\quad$ Dominant) & & & & \\
0.322 & 1.023 & 1.771 & 2.198 & 2.289 \\
0.447 & 7.965 & 8.106 & 9.545 & 10.809 \\
1.137 & 17.804 & 4.982 & 38.499 & 54.145 \\
\hline
\end{tabular}

and $[1.90 \mathrm{~s}, 2.65 \mathrm{~s}, 0.47 \mathrm{~s}, 1.99 \mathrm{~s}, 988]$ at bus 34. From simulation, no FIDVR is observed and the average absolute speed deviation of all generators is further reduced to $0.0406 \mathrm{~Hz}$.

\subsection{Comparison results}

Remarks Tables 2 and 3 and Fig. 7, compare the above four scenarios named "No SVC", "SVC-default", "SVC-1 OPT", and "SVC-3 OPT", respectively for the scenario without SVCs, the scenario with 3 SVCs using the default parameters, the scenario with $3 \mathrm{SVCs}$ using the same set of

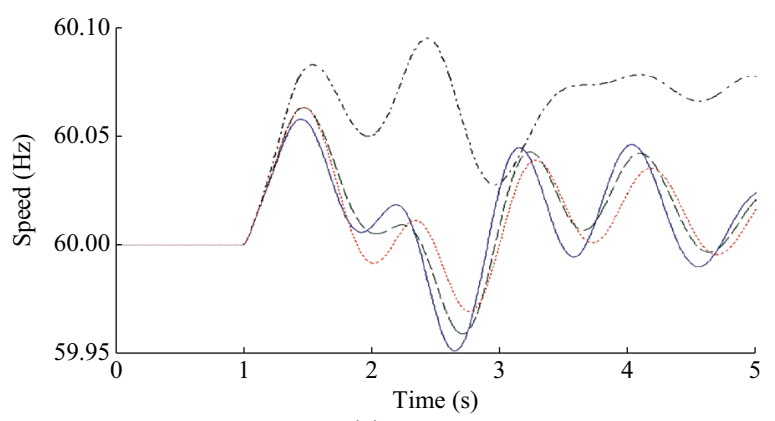

(a) Generator 47

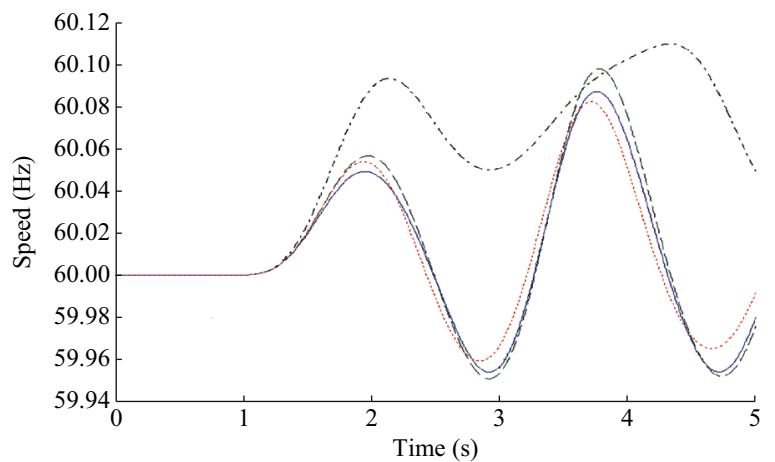

(b) Generator 91

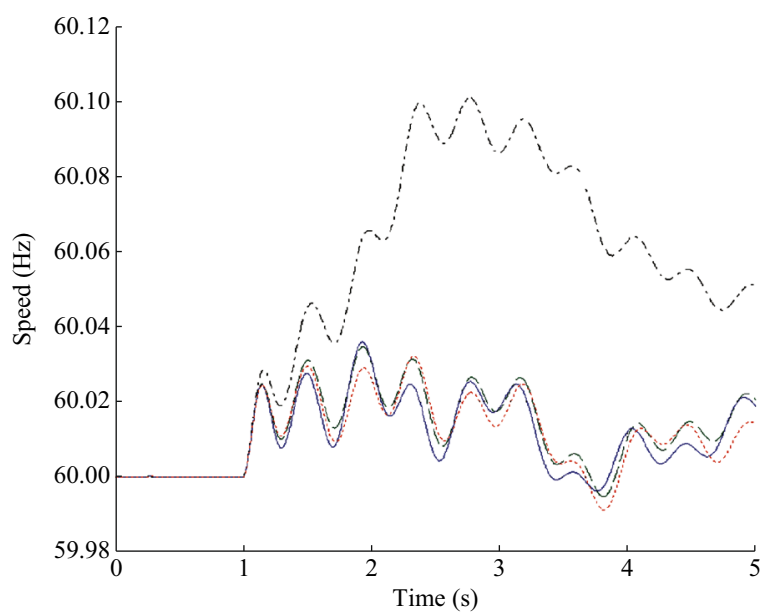

(c) Generator 130

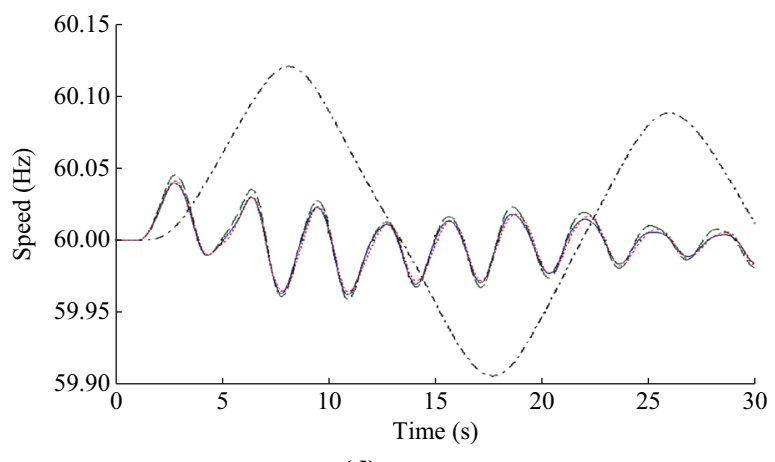

(d) Generator 139

-- No SVC; -... SVC-default; - - SVC-1 OPT; - SVC-3 OPT

Fig. 7 Comparison of speed of typical generators 
optimized controller parameters, and the scenario with 3 SVCs using respectively optimized controller parameters.

From Table 2, the objective function, i.e. the average absolute speed deviation of all generators, gradually decreases from "No SVC" to "SVC-3 OPT", which is reflected from Fig. 7 by comparing the speeds of four representative generators at buses 47, 91, 130, and 139 .

From Fig. 7, results of the scenarios using the default parameters, using one optimized set of parameters for all SVCs, and using three respectively optimized sets of parameters do not have huge differences. However, slight improvements in the post-contingency waveforms may still significantly reduce the variations in active power flows in the power network so as to bring economic benefits.

Oscillation modes and their corresponding damping ratios can be estimated from the waveforms of post-fault generator speeds. Based on the prony analysis, the dominant inter-area mode has frequency $0.573 \mathrm{~Hz}$. The two groups of oscillating generators are colored differently in Fig. 3. The purple generators oscillate against the pink generators. The generators with high participant factors are indicated by brown arrows. Specifically, generators 22, 23, 24, and 25 are against generators 91, 92, 97 and 98. Without any SVC, the damping ratio of this mode is $3.229 \%$. With three SVCs, its damping ratio is observed to increase to $6.703 \%, 8.406 \%$, and $11.088 \%$ respectively for scenarios "SVC-default", "SVC-1 OPT", and "SVC-3 OPT".

Besides the dominant mode at $0.573 \mathrm{~Hz}$, Table 3 also compares other three modes for four scenarios. It can be observed that "SVC-3 OPT" results in the best damping ratios than any other scenarios and "SVC-1 OPT" is the second one. Most of damping ratios under the "SVC-default" scenario are better than those under the "No SVC" scenario except for that with $1.137 \mathrm{~Hz}$ mode, which is caused by the sacrifice of oscillation damping to support voltage responses. Increased damping ratios of the aforementioned four modes reflect the improvement of postfault angular stability.

In summary, angular stability can significantly be improved, too, by the SVCs installed to prevent FIDVR issues using the SVC controller parameters optimized by the proposed method. Here, the angular stability mainly concerns the oscillation damping and speed deviations of the system following the most critical " $N-1$ " contingency. The proposed method is capable of optimizing either one set of five controller parameters for all SVCs or respective sets of parameters for different SVCs.

\section{Conclusion}

This paper proposes an MADS-based blackbox optimization approach for the optimal control parameters of SVCs, which efficiently interfaces a power system DAE solver with the MADS algorithm. The proposed approach can assist power system planning engineers to optimize the control parameters of several installed SVCs, which not only mitigates FIDVR issues but also improves angular stability. This is an important and time-consuming task at many electric utilities, especially those concerning angular stability along with potential FIDVR issues. The proposed approach has been successfully validated on a detailed NPCC system model with realistic load models.

Acknowledgements This work was supported in part by the ERC Program of the NSF and DOE under NSF Grant EEC-1041877.

Open Access This article is distributed under the terms of the Creative Commons Attribution 4.0 International License (http:// creativecommons.org/licenses/by/4.0/), which permits unrestricted use, distribution, and reproduction in any medium, provided you give appropriate credit to the original author(s) and the source, provide a link to the Creative Commons license, and indicate if changes were made.

\section{References}

[1] ABB (2015) SVC, an insurance for improved grid system stability and reliability. https://library.e.abb.com/public/ 75362d2c1aa7f86783257e0c00478a6f/SVC\%20A02-0100.pdf. Accessed 30 August 2017

[2] Tiwari A, Ajjarapu V (2016) Addressing short-term voltage stability problem Part II: case study. In: Proceedings of IEEE PES transmission and distribution, Dallas, USA, 3-5 May 2016, $5 \mathrm{pp}$

[3] Meliopoulos SA, Vittal V, McCalley JD et al (2008) Optimal allocation of static and dynamic VAR resources. Dissertation, Arizona State University

[4] Sapkota B, Vittal V (2010) Dynamic var planning in a large power system using trajectory sensitivities. IEEE Trans Power Syst 25(1):461-469

[5] Krishnan V, Liu H, McCalley J (2009) Coordinated reactive power planning against power system voltage instability. In: Proceedings of IEEE PES power systems conference and exposition, Seattle, USA, 15-18 March 2009, 8 pp

[6] Qi J, Huang W, Sun K et al (2017) Optimal placement of dynamic var sources by using empirical controllability covariance. IEEE Trans Power Syst 32(1):240-249

[7] Tiwari A, Ajjarapu V (2011) Optimal allocation of dynamic VAR support using mixed integer dynamic optimization. IEEE Trans Power Syst 26(1):305-314

[8] Paramasivam M, Salloum A, Ajjarapu V et al (2013) Dynamic optimization based reactive power planning to mitigate slow voltage recovery and short term voltage instability. IEEE Trans Power Syst 28(1):3865-3873

[9] Xu Y, Dong Z, Meng K et al (2014) Multiple-objective dynamic var planning against short-term voltage instability using a 
decomposition-based evolutionary algorithm. IEEE Trans Power Syst 29(6):2813-2822

[10] Wildenhues S, Rueda J, Erlich I (2015) Optimal allocation and sizing of dynamic var sources using heuristic optimization. IEEE Trans Power Syst 30(56):2538-2546

[11] Liu H, Krishnan V, McCalley JD et al (2014) Optimal planning of static and dynamic reactive power resources. IET Gener Transm Distrib 8(12):1916-1927

[12] Hussain Z, Chen Z, Thogersen P et al (2015) Dynamic reactive power compensation of large-scale wind integrated power system. IEEE Trans Power Syst 30(5):2516-2526

[13] Huang W, Sun K, Qi J et al (2014) A new approach to optimization of dynamic reactive power sources addressing FIDVR issues. In: Proceedings of IEEE PES general meeting, National Harbor, USA, 27-31 July 2014, 5 pp

[14] Huang W, Sun K, Qi J et al (2015) Voronoi diagram based optimization of dynamic reactive power sources. In: Proceedings of IEEE PES general meeting, Denver, USA, 26-30 July 2015, $5 \mathrm{pp}$

[15] Zhao Q, Jiang J (1994) Robust SVC controller design for improving power system damping. IEEE Trans Power Syst 10(4):1927-1932

[16] Mithulananthan N, Cañizares CA, Reeve J et al (2003) Comparison of PSS, SVC and STATCOM controllers for damping power system oscillations. IEEE Trans Power Syst 18(2):786-792

[17] Wang L, Truong D (2013) Stability enhancement of a power system with a PMSG-based and a DFIG-based offshore wind farm using a SVC with an adaptive-network-based fuzzy inference system. IEEE Trans Power Syst 60(7):2799-2807

[18] Zhang S, Vittal V (2013) Design of wide-area power system damping controller resilient to communication failures. IEEE Trans Power Syst 28(4):4292-4300

[19] Yao W, Jiang L, Wen J et al (2014) Wide-area damping controller of FACTS devices for inter-area oscillations considering communication time delay. IEEE Trans Power Syst 29(1):318-329

[20] Bian XY, Geng Y, Lo KL et al (2016) Coordination of PSSs and SVC damping controller to improve probabilistic small signal stability of power system with wind farm integration. IEEE Trans Power Syst 31(3):2371-2382

[21] Vahidnia A, Ledwich G, Palmer EW (2016) Transient stability improvement through wide-area controlled SVCs. IEEE Trans Power Syst 31(4):3082-3089

[22] Fetouh T, Zaky MS (2017) New approach to design SVC-based stabilizer using genetic algorithm and rough set theory. IET Gener Transm Distrib 11(2):372-382

[23] Shahgholian G, Movahedi A (2016) Power system stabilizer and flexible alternating current transmission systems controller coordinated design using adaptive velocity update relaxation particle swarm optimization algorithm in multi-machine power system. IET Gener Transm Distrib 10(8):1860-1868

[24] Digabel SL (2011) NOMAD: nonlinear optimization with the MADS algorithm. ACM Trans Math Softw 37(4):44

[25] Audet C, Dennis JE (2006) Mesh adaptive direct search algorithms for constrained optimization. SIAM J Optim 17(1):188-217

[26] Qi J, Sun K, Kang W (2015) Optimal PMU placement for power system dynamic state estimation by using empirical observability gramian. IEEE Trans Power Syst 30(4):2041-2054

[27] Huang W, Sun K, Qi J et al (2017) Optimization of dynamic reactive power sources using mesh adaptive direct search. IET Gener Transm Distrib 11(15):3675-3682

[28] Ali M, Khompatraporn C, Zabinsky Z (2005) A numerical evaluation of several stochastic algorithms on selected continuous global optimization test problem. J Glob Optim 31(4):635-672

[29] Huang W, Sun K, Qi J et al (2017) Optimal allocation of dynamic var sources using Voronoi diagram method integrating linear programming. IEEE Trans Power Syst 32(6):4644-4655

[30] Siemens PTI Power Technologies Inc., PSS/E 33 (2011) Program application guide II

Weihong HUANG received the B.E. degree in electrical engineering from Huazhong University of Science and Technology, Wuhan, China, in 2009, and the master degree as well as Ph.D. degree from University of Tennessee, Knoxville, USA in 2014 and 2017, both in electrical engineering. She worked at Yueqing Electric Power Bureau, Grid State Corporation of China, Wenzhou, China, from 2009 to 2012. Currently, she is a planning engineer in New York Independent System Operator, Rensselaer, USA. Her research interests include planning and control of reactive power and model validation.

Kai SUN received the B.S. degree in automation in 1999 and the Ph.D. degree in control science and engineering in 2004 both from Tsinghua University, Beijing, China. He is currently an associate professor at the Department of EECS, University of Tennessee, Knoxville, USA. He was a project manager in grid operations and planning at the EPRI, Palo Alto, from 2007 to 2012. He serves in the editorial boards of IEEE Transactions on Smart Grid, IEEE Access, IET Generation, Transmission and Distribution, and Journal of Modern Power Systems and Clean Energy. He is a recipient of 2016 NSF CAREER award. His research interests include stability, dynamics and control of power grids and other complex systems. 